\title{
The Study of Robot Movement Inverse Solution Based on Genetic
} Algorithm

\author{
Shen $\mathrm{Chao}^{1}$ \\ ${ }^{1}$ University of Shanghai for Science and Technology, Shanghai, China \\ Correspondence: Shen Chao, College of Mechanical Engineering, University of Shanghai for Science and \\ Technology, 334th, Jun Gong Road, Shanghai 200093, China. Tel: 86-137-6112-1824. E-mail: \\ petershen88@126.com
}

Received: March 2, 2013

Accepted: April 24, $2013 \quad$ Online Published: May 14, 2013

doi:10.5539/mas.v7n6p53

URL: http://dx.doi.org/10.5539/mas.v7n6p53

\begin{abstract}
Through the analysis of three degree of freedom of the industrial robot bar geometric parameter, the homogeneous transformation matrix method was used to establish the system of positive kinematics model. This paper will introduce genetic algorithm to solve the problem of inverse kinematics robot to plane three degree of freedom robot, to explain the genetic algorithm is applied to solve the inverse kinematics more effectively.
\end{abstract}

Keywords: robot, forward and inverse kinematics, genetic algorithm

\section{Introduction}

Based on the robot kinetic theory (Yu \& Ma, 1999), we establish the robot kinematic model. According to the forward kinematics theory, under the conditions of known geometric parameters and the joint angle of the lever member, the position and attitude of the reference coordinate system relative to the manipulator end effector (Ma, 1991). Based on genetic algorithm, we put forward a new kind of algorithm controlling robot pose, and solve the inverse kinematics problem.

\section{Establishment of Robot System Composition and Kinematics Model}

\subsection{Robot Kinematics Introduction (Jiang, 1994)}

Control of robot kinematics model (Chen \& Zhang, 2005) is divided into two parts, forward kinematics and inverse kinematics. Solving the forward kinematics problem (Guo \& Wang, 2012), a general method in engineering is homogeneous matrix transform method, presented by Denavit and Hartenberg. The core idea is build homogeneous transformation matrix for each robot joint rod, through successive matrix operations, which will use the robot manipulator end effector coordinate system representation of the vector to the base coordinate system. And the establishment of the corresponding relationship between the robot joint variables and rod of homogeneous coordinates.

\subsection{Establishment of Robot Kinematics Model}

In order to describe the joint movement, the position and attitude of the reference coordinate system relative to the manipulator end effector, according to D-H member axes rule, each robot each rod in the joint shaft is set up by regular Descartes attached body coordinate system $\left(x_{i}, y_{i}, z_{i}\right)$, where $i=1,2, \ldots, n(n$ is the number of degrees of freedom), as Figure 1 shown below. As long as the $\mathrm{z}_{0}$ axis along the first joint motion axis, the position and direction of the manipulator at 0 system on the base can be optionally. As long as the $x_{n}$ axis and $z_{i-1}$ axis is vertical, the last coordinates $\left(\mathrm{x}_{\mathrm{n}}, \mathrm{y}_{\mathrm{n}}, \mathrm{z}_{\mathrm{n}}\right)$ can be placed in any position of the manipulator end. When the joint $\mathrm{i}$ movement, rod i relative to the rod member $\mathrm{i}_{\mathrm{i}-1}$ motion, on which the coordinates $\left(\mathrm{x}_{\mathrm{i}-1}, \mathrm{y}_{\mathrm{i}-1}, \mathrm{z}_{\mathrm{i}-1}\right)$ relative to the rod member $\mathrm{i}-1$ will also move, the coordinates $\left(\mathrm{x}_{\mathrm{i}-1}, \mathrm{y}_{\mathrm{i}-1}, \mathrm{z}_{\mathrm{i}-1}\right)$ relative to the rod I-1 coordinate system $\left(\mathrm{x}_{\mathrm{i}-2}, \mathrm{y}_{\mathrm{i}-2}, \mathrm{z}_{\mathrm{i}-2}\right)$ will also move. 


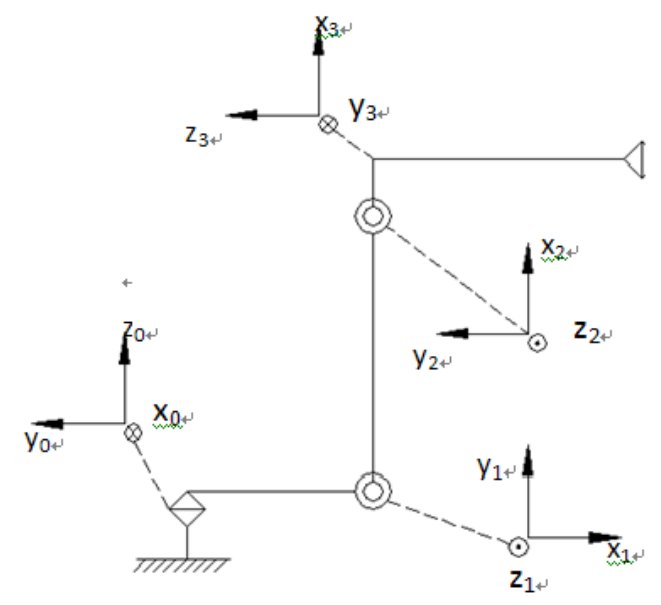

Figure 1. Robot link coordinate system

Based on the robot pole coordinate system, denoted by D-H method can be used to calculate the homogeneous transformation matrix connecting i coordinates and i-1 coordinates, a point $\mathrm{P}_{\mathrm{i}}$ represented by icoordinate can be available through coordinates $\mathrm{i}-1$, just accomplished the transformation one by one:

(1) $\mathrm{x}_{\mathrm{i}-1}$ axis around the $\mathrm{z}_{\mathrm{i}-1}$ axis angle $\theta_{\mathrm{i}}$, making it the same $\mathrm{x}_{\mathrm{i}}$ axis alignment;

(2) move along the $\mathrm{z}_{\mathrm{i}-1}$ axis translation distance $\mathrm{d}_{\mathrm{i}}$, the $\mathrm{x}_{\mathrm{i}-1}$ axis and $\mathrm{x}_{\mathrm{i}}$ axis coincide;

(3) move along the $x_{i}$ axis distance $a_{i}$, icoordinates and $i-1$ coordinates of the origin and the $x$ axis coincide;

(4) around the $x_{i}$ axis angle of $i, i$ coordinates and $i-1$ coordinates coincide completely.

With basic homogeneous rotation-translation matrix representation and multiplicative quadature of these four kind of action, we'll obtain the adjacent coordinates $\mathrm{i}$ and $\mathrm{i}-1$ synthesis homogeneous transformation matrix $\mathrm{A}_{\mathrm{i}}$ : ${ }^{\mathrm{i}-1} \mathrm{~A}_{\mathrm{i}}=\operatorname{Rot}\left(\mathrm{z}, \theta_{\mathrm{i}}\right) \operatorname{Trans}\left(0,0, \mathrm{~d}_{\mathrm{i}}\right) \operatorname{Trans}\left(\mathrm{a}_{\mathrm{i}}, 0,0\right) \operatorname{Rot}\left(\mathrm{x}, \mathrm{a}_{\mathrm{i}}\right)$

Expand the above equation can be obtained:

$$
{ }^{\mathrm{i}-1} \mathrm{~A}_{\mathrm{i}}=\left[\begin{array}{cccc}
\cos \theta_{i} & -\cos \alpha_{i} \sin \theta_{i} & \sin \alpha_{i} \sin \theta_{i} & \alpha_{i} \cos \theta_{i} \\
\sin \theta_{i} & \cos \alpha_{i} \cos \theta_{i} & -\sin \alpha_{i} \cos \theta_{i} & \alpha_{i} \sin \theta_{i} \\
0 & \sin \alpha_{i} & \cos \alpha_{i} & d_{i} \\
0 & 0 & 0 & 1
\end{array}\right]
$$

When the mechanical hand of each link coordinates were specified, will be able to list constant parameters in all links. The rotation joint, $\mathrm{a}_{\mathrm{i}}$, $\mathrm{i}$ and $\mathrm{d}_{\mathrm{i}}$ is a constant, $\theta_{\mathrm{i}}$ is the joint variables.

Using ${ }^{i-1} A_{i}$ matrix, the robot arm on a fixed point $P_{i}$ in the $i$ coordinate system of homogeneous coordinates and i-1 coordinates in the homogeneous coordinates of link, have equation:

$$
\mathrm{P}_{\mathrm{i}-1}=\left(\mathrm{x}_{\mathrm{i}-1}, \mathrm{y}_{\mathrm{i}-1}, \mathrm{z}_{\mathrm{i}-1}, 1\right)^{\mathrm{T}}, \mathrm{Pi}=\left(\mathrm{x}_{\mathrm{i}}, \mathrm{y}_{\mathrm{i}}, \mathrm{z}_{\mathrm{i}}, 1\right)^{\mathrm{T}} \text {. }
$$

Establishment of robot forward kinematics model, as shown below:

The table shows the link parameters, each connecting transformation matrix can be obtained as follows:

$$
{ }^{0} T_{1}=\left[\begin{array}{cccc}
c \theta_{1} & -s \theta_{1} & 0 & 330 c \theta_{1} \\
s \theta_{1} & c \theta_{1} & 0 & 330 s \theta_{1} \\
0 & 0 & 1 & 0 \\
0 & 0 & 0 & 1
\end{array}\right] \quad{ }^{1} T_{2}=\left[\begin{array}{cccc}
-s \theta_{2} & -c \theta_{2} & 0 & -300 s \theta_{2} \\
c \theta_{2} & s \theta_{2} & 1 & 300 c \theta_{2} \\
0 & 0 & 0 & 0 \\
0 & 0 & 0 & 1
\end{array}\right] \quad{ }^{2} T_{3}=\left[\begin{array}{cccc}
s \theta_{3} & c \theta_{3} & 0 & 150 s \theta_{3} \\
-c \theta_{3} & s \theta_{3} & 0 & -150 c \theta_{3} \\
0 & 0 & 1 & 0 \\
0 & 0 & 0 & 1
\end{array}\right]
$$

Wherein, ${ }^{0} \mathrm{~T}_{1}$ said connecting rod 1 coordinate $\{1\}$ relative to the pose of base coordinate system $\{0\},{ }^{1} \mathrm{~T}_{2} 、{ }^{2} \mathrm{~T}_{3}$ and so on, $\mathrm{c} \theta_{1}, \mathrm{~s} \theta_{1}$ represent $\sin \theta_{1}, \cos \theta_{1}$, and the rest of the analog.

Design of manipulator end pole coordinate system to the robot coordinate system homogeneous transformation matrix, then the 


$$
{ }^{0} T_{3}={ }^{0} A_{1}{ }^{1} A_{2}{ }^{2} A_{3}
$$

Any of the known robot joint angle vector substituting into the above formula, we can find the homogeneous coordinates values of robot manipulator end relative to the respective reference coordinate system. Establish the corresponding relationship between robot end-effector position and attitude and the joint variable value. In the robot joint constraints, given any set of joint variables, through the forward kinematics equation uniquely determine the end-effector pose.

\section{Robot Gripping Control Algorithm}

On the basis of establishment of robot kinematic model in the above context, this chapter is based on the genetic algorithm robot gripping control algorithm, analyze solution of the robot inverse kinematics, illustrate specific implementation process about genetic algorithmic and a planar robot with three degrees of freedom.

\subsection{Inverse Kinematics of the Robot}

The robot kinematics equation can be obtained from the equations of robot motion:

$$
{ }^{0} \mathrm{~T}_{3}=\left[\begin{array}{cccc}
n_{x} & x_{x} & a_{x} & p_{x} \\
n_{y} & O_{y} & a_{y} & p_{y} \\
n_{z} & \sigma_{z} & a_{z} & p_{z} \\
0 & 0 & 0 & 1
\end{array}\right]={ }^{0} T_{1}\left(\theta_{1}\right)^{1} T_{2}\left(\theta_{2}\right)^{2} T_{3}\left(\theta_{3}\right)
$$

If the robot end effector connecting rod pose has been given, i.e. $\mathrm{n}, \mathrm{o}$, a and $\mathrm{p}$ were known, then seek the joint variables $\theta_{1}, \theta_{2}, \theta_{3}$, the value of them is called the inverse solution.

Robot inverse kinematics problem is when the position, attitude, velocity, angular velocity, acceleration and angular acceleration of the robot end effector rod are presented, and can achieve these requirements of the joint position, velocity and acceleration. This article only discusses the problem in the first place, which is known as satisfy some work requirements of the spatial position and posture A of end effector, and each pole structure parameters, and the robot joint variables can be calculated.

\subsection{Genetic Algorithm}

Genetic algorithm referred to as GA, is a kind of evolutionary algorithm first proposed by the United States of America professor J. H. Holland in 60s later period, it's the most widely used. Genetic algorithm is a kind of optimization search algorithm draw from biological natural election and natural genetic mechanisms, is an approximation algorithm.

Genetic algorithm (Zhang, 1995) the term is derived from the nature genetics. An alternative solution is called a chromosome, each chromosome consists of several genes, each gene can be used to represent a number, a number of chromosomes compose a group. Genetic algorithm (Wang \& Wei, 1996) is an alternative solution for iterative computation process, and each iteration called generation. At the completion of iteration, the current group will make use of the certain evaluation function performance evaluation, and on the basis of the evaluation of new generation. Initial groups randomly determined to subjective experience, groups of fixed capacity for $\mathrm{N}$, specific algorithm is shown by the following:

1) $\mathrm{t}=0$;

2) generate an initial group of $G(t)$;

3) with a certain evaluation function to evaluate $G(t)$;

4) satisfy the end condition, transferred to 8$)$;

5) produce a new generation of $\mathrm{G}(\mathrm{t}+1)$ by $\mathrm{G}(\mathrm{t})$;

6) to evaluate $G(t+1)$;

7) back to 4);

8) stop;

The above iterative compute process to find a satisfying solution or to preset number of iterations. Algorithm of the evaluation function is mainly used to evaluate each of the chromosomes in the current generation, by evaluating the next generation of removed a number of low performance of chromosomes, keep some high-performance chromosomes, and through genetic operators added some new chromosomes, to ensure that the next generation of group contains new information, make its average performance is improved, the result was 
very good, to meet the requirements of the problem solving.

In general, GA is not a simple optimization algorithm, it is not a traditional deterministic calculation tool, but it is a kind of new optimization algorithm that based on the evolutionary ideas, and it's a powerful tool to solve the problem of large-scale complex.

\subsection{Planar Robot Example}

One, parameters of robot connecting links.

Take a three planar robot as an example here. Link parameters are shown in Table 1.

Table 1. The three connecting rod mechanical parameters

\begin{tabular}{ccccc}
\hline Angle & $\alpha_{i-1}$ & $\theta_{i}$ & $l_{i}$ & $\alpha_{i}$ \\
\hline 1 & 0 & $\theta_{l}$ & $l_{l}$ & 0 \\
2 & 0 & $\theta_{2}+90$ & $l_{2}$ & 0 \\
3 & 0 & $\theta_{3}-90$ & $l_{3}$ & 0 \\
\hline
\end{tabular}

Two, the derivation of end effector position.

According to the link parameter definition can get transformation matrix:

$$
{ }^{i-1} A_{i}=\left[\begin{array}{cccc}
c_{i} & -s_{i} & 0 & a_{i-1} \\
s_{i} & c_{i} & 0 & 0 \\
0 & 0 & 1 & 0 \\
0 & 0 & 0 & 1
\end{array}\right]
$$

The position matrix of end effector:

$$
{ }^{0} T_{3}={ }^{0} T_{1}{ }_{1}^{1} T_{2}{ }^{2} T_{3}{ }^{3} T_{E}=\left[\begin{array}{cccc}
c_{123}-s_{123} & 0 & c_{123} l_{3}+c_{12} l_{2}+c_{1} l_{1} \\
s_{123} & c_{123} & 0 & s_{123} l_{3}+s_{12} l_{2}+s_{1} l_{1} \\
0 & 0 & 1 & 0 \\
0 & 0 & 0 & 1
\end{array}\right]
$$

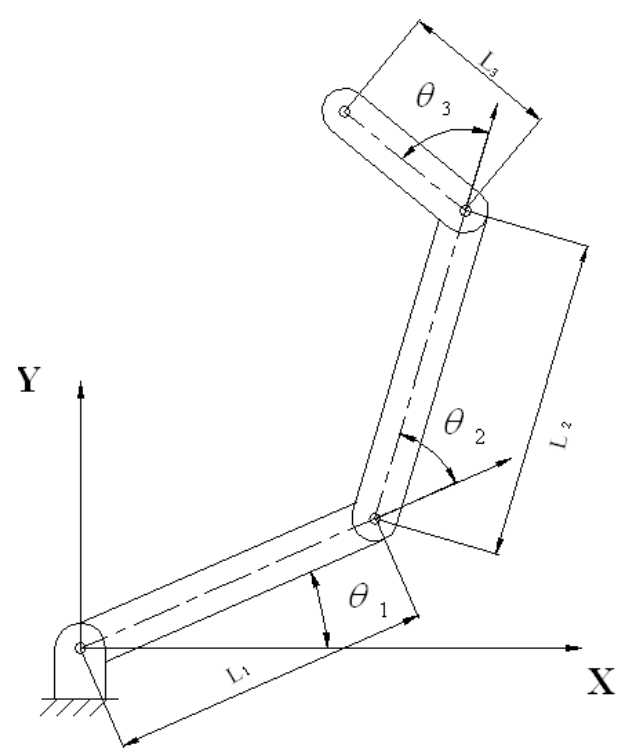

Figure 2. Diagram of three lever operating machine

At the end of three lever operating machine, the $\mathrm{X}$, y coordinates can be expressed as: 


$$
\begin{aligned}
& x=l_{1} \cos \theta_{1}+l_{2} \cos \left(\theta_{1}+\theta_{2}\right)+l_{3} \cos \left(\theta_{1}+\theta_{2}+\theta_{3}\right) \\
& y=l_{1} \sin \theta_{1}+l_{2} \sin \left(\theta_{1}+\theta_{2}\right)+l_{3} \sin \left(\theta_{1}+\theta_{2}+\theta_{3}\right)
\end{aligned}
$$

The parameters of an operating machine of the rod respectively is $1_{1}=330,1_{2}=300,1_{3}=150$, firstly, the control parameters of genetic algorithm are as follows: group capacity N, genetic algebra G, crossover probability C, mutation probability $\mathrm{M}$, according to the number of variables, determine the chromosome number of 3 , vector situation expressed as $\left[\theta_{1}, \theta_{2}, \theta_{3}\right]$ range of each variable is $\left[-180^{\circ}, 180^{\circ}\right]$, in the absence of prior knowledge $\theta_{1}, \theta_{2}$, $\theta_{3}$ in their range, random generate chromosome $\mathrm{N}\left[\theta_{11}, \theta_{21}, \theta_{3 \mathrm{i}}\right], \mathrm{i}=1,2, \ldots, \mathrm{N}$, as the initial population.

Selection of genetic parameter control:

As the above genetic algorithm parameter selection, we know, population capacity of $\mathrm{N}$ affects the final characteristics and effectiveness of genetic algorithm. Small group capacity will make the convergence speed slow, but the large group capacity will increase computing workload of each generation, and when $\mathrm{N}$ greater than 100 , the convergence of the improvement is not obvious, therefore recommend selection $\mathrm{N}=100$.

Crossover probability determined the crossover frequency, large crossover probability means that each generation will have more new chromosome. Recommend $\mathrm{C}=0.9$.

The mutation probability determines the probability of mutation operator.

Too large Mutation probability will lead to a good chromosome loss of information. While too small mutation probability will do no help to the improvement of the convergence of the algorithm. Generally it takes $\mathrm{M}=0.03-0.07$.

$\mathrm{N}=100, \mathrm{C}=0.9, \mathrm{M}=0.04$, genetic end condition (Shi, Chen, \& Yao, 2009) is:

$$
J=\sqrt{\left(x-x_{0}\right)^{2}+\left(y-y_{0}\right)^{2}}<0.1
$$

Take the position coordinates of the end effector $(651,421)$, and then follow the above genetic algorithm genetic operation, until it meets the end condition. Finally the minimum of the group J chromosomes is considered as each rod corner solutions for robot mechanical hands.

In the solution of genetic algorithm in this paper, in order to avoid the loss of information good chromosome, during each generation of genetic, ten optimal chromosome were selected and retained to the new generation, this also ensures that the performance of a new generation is at least better than that in later generation. In addition, mutation operation method has influence on convergence speed. In order to improve the convergence speed of the algorithm, the selected gene mutation in genetic will initially replace its value of $110 \%$ or $90 \%$ of the current number (probability 50\%), after each increase of 10 generations, the changes in the value of a corresponding reduction in 0.99 or 1.01 times, this makes the search range from big to small, especially with the genetic algebra increases, the method helps to accelerate the search speed, when search performance cannot be improved significantly.

The calculation results are as follows:

The G 100 generation, $=0.54$;

The $\mathrm{G} 150$ generation, $=0.092$.

As can be seen the condition $(*)$, from this group, J value of the smallest chromosome is regarded as the desired solution, then the manipulator end-effector position coordinates is $(651.06,421.07)$, close to the target value $(651$, 421).

\section{Conclusions}

According to definition of robot bar geometric parameters, analyze link parameters in three degree of freedom industrial robot, using homogeneous transformation matrix method to set up the robot forward kinematics model.

Genetic algorithm is used to solve the inverse kinematics. The robot kinematics equation is highly nonlinear and strong coupling equations, the traditional optimization method for kinematic solving has two problems: firstly, it is too complex, algorithm efficiency is low; secondly, it is easy to fall into local optimization, it is difficult to find the global optimal solution. In this paper, genetic algorithm is introduced into the solution of inverse kinematics problem, proposed using real number coding to improve the computing efficiency of the algorithm and accuracy. With an example of the plane robot which has three degrees of freedom illustrate the application of genetic algorithm to the validity of robot inverse k-inematics solution. Genetic algorithm constitute a new intelligent control method, the research in robotics has opened up a broad field, also it is bound to the 
development of intelligent robots.

\section{References}

Chen, L. P., \& Zhang, Y. Q. (2005). Dynamics analysis of mechanical system. Beijing: Tsinghua university press.

Guo, Y. K., \& Wang, F. Z. (2012). The analysis and comparison of regional pole assignment in the robot movement. Computer tehnology and automation, 31(4), 21-25.

Jiang, X. F. (1994). Introduction of robotics. pp. 221-229. Shenyang: Liaoning Science and Technology Press.

Ma, X. F. (1991). Robot mechanism (1st ed.). Beijing: Mechanical Industry Press.

Shi, L., Chen, T. J., \& Yao, L. N. (2009). Theory and application of Intelligent control. Shanghai Jiaotong University Press, 4(1), 300-303.

Wang, H. J., \& Wei, X. F. (1996). Genetic algorithm Overview. Control theory and applications, 13(6), 697-707.

Yu, D. T., \& Ma, X. F. (1999). Application of the industrial robot engineering. Beijing: Metallurgical Industry Press.

Zhang, Y. Q. (1999). Genetic optimization techniques for beginners. Journal of Kunming University of Science and Technology, 24(3), 41-45 